\title{
Annual \\ acknowledgment \\ of manuscript \\ reviewers
}

Eye (2013) 27, 1335-1338; doi:10.1038/eye.2013.241

In this issue, we acknowledge and thank the reviewers who have been active over the past year. We greatly appreciate their tireless and largely unseen work. It is thanks to them that the quality of papers continues to increase.

Editor: Andrew J Lotery

Top 10 reviewers

Ahfat, FG

Alaghband, Pouya

Allan, Bruce

Amoaku, Winfried

Eye Reviewer Thank You List 2013
Gerendas, Bianca S

Hirst, Lawrence W

Kheirkhah, Ahmad

Sugar, Alan
Sugar, Joel

Vavvas, Demetrios G

\section{Abbott, Joe}

Abcouwer, Steven F

Abdalla, Ebtesam

Abdelrahman, Ahmed M

Abdul Karim, Mohamed Naved

Abramoff, Michael D

Acharya, Nachiketa

Acheson, James

Aclimandos, Wagih

Adams, Gillian G

Agrawal, Ashish

Agrawal, Pallavi

Agrawal, Shishir

Ahfat, FG

Ahluwalia, Harpreet S

Akpek, Esen

Alaghband, Pouya

Alapure, Bhagwat V

Allan, Bruce

Allen, David

Amoaku, Winfried

Amparo, Francisco

Anand, Nitin

Anderson, David F

Andreoli, Christopher

Ang, Alan

Ang, Ghee Soon

Ansari, Ejaz

Ansari, Imran

Antcliff, Richard

Aptel, Florent

Arevalo, J Fernando

Arias, Luis

Armitage, John

Arnold, Anthony
Arora, Rashi

Arshinoff, Steve A

Ashok Kumar, Dhivya

Ashworth, Jane

Ataullah, Sajid

Atherley, Carolyn

Atkinson, Peter L

Aung, Tin

Azad, Raj V

Babu, Kalpana

Baile, Rahul B

Bailey, Christopher S

Bakhtiari, Pejman

Balasubramanian, Madhusudhanan

Ball, James L

Bandello, Francesco

Banerjee, Somnath

Banker, Alay S

Bansal, Reema

Baraas, Rigmor C

Baradaran ebrahimi, Katayoon

Barton, Keith

Bastawrous, Andrew

Bates, Richard

Batmanabane, Vaishnavi Prakash

Battu, Rajani

Beare, NA

Beatty, Stephen

Beck, Allen

Beigi, Bijan

Beit-Yannai, Elie

Bell, Katharina

Ben Simon, Guy J

Berry, Monica
Best, Stephen

Bhende, Pramod S

Biswas, Jyotirmoy

Biswas, Susmito

Black, Graeme CM

Blinder, Kevin J

Boddu, Sai Sagar

Bodis-Wollner, Ivan

Boldin, Ingrid

Bourges, Jean-Louis

Bourne, Rupert R

Brand, Christopher

Brazier, John

Broadway, David C

Brodie, Scott

Bron, Alain M

Bron, Anthony J

Brookes, John

Brusini, Paolo

Buckley, Roger

Budenz, Donald

Bunce, Catey

Burke, Tomas

Burton, Ben

Burton, Robert L

Busin, Massimo

Butler, Thomas K

Caesar, Ric

Callear, Andrew

Callejo, Sonia A

Carpenter, John

Carvounis, Petros E

Cauchi, Paul

Cavalcanti, Bernardo
Cekic, Osman

Cennamo, Giovanni

Cestari, Dean

Chakrabarti, Subhabrata

Chalam, Kakarla V

Challa, Pratap

Chan, Carmen K

Chan, Choi M

Chan, Clement K

Chan, Colin C

Chan, Jane W

Chan, Jonathan $\mathrm{CH}$

Chanana, Bhuvan

Chandra, Parijat

Chang, Andrew

Chang, Bernard Y

Chapman, Fiona

Charbel Issa, Peter

Charles, Stephen

Charles, Steve

Chattopadhyay, Abhijit

Chatziralli, Irini P

Chen, Celia S

Chen, Fred K

Chen, Haoyu

Chen, Lei

Chen, Li Jia

Chen, Shih-Jen

Chen, Simon D

Chen, Xiaoli

Chen, Yanjun (Judy)

Cheng, Ching-Yu

Cheung, Carol

Cheung, David

Chiang, Mark Y 
Chisholm, Iain $\mathrm{H}$

Chiu, Chung-Jung

Cho, Hee-Yoon

Chodosh, James

Chow, David

Chua, Chung N

Cillino, Salvatore

Ciralsky, Jessica

Claoue, Charles

Clare, Gerald

Clarke, Jonathan C

Clarke, Michael

Cockerham, Glenn C

Cohen, Kenneth

Cohen, Salomon Yves

Comyn, Oliver

Cook, Stuart

Cottrell, David G

Coupland, Sarah E

Crabb, David

Cunha-Júnior, Armando S

Cunningham, Jr, Emmett T

Currie, Zanna

Cuthbertson, Fiona M

Daly, Mary K

Damato, Bertil

Dapling, Robert

Davies, Nigel

Dayan, Colin

De La Cruz, Jose

Deane, James S

Denniston, Alastair

Devonport, Helen

Dietlein, Thomas S

Dimitrova, Galina

Dinakaran, Subramanian

Ding, Xiaoyan

Djalilian, Ali

Dogru, Murat

Dolman, Peter

Downey, Louise

Dsouza, Yvonne

Duggal, Priya

Dupps, William J

Durukan, Ali H

Eckstein, Mike

Edelhauser, Henry

Edmunds, Beth

El Hindy, Nabil

Elliott, Andrew

Elsheikh, Ahmed

Ezra, Daniel

Fauser, Sascha

Feke, Gilbert T

Ferreras, Antonio

Fingert, John

Flaxel, Christina

Fleck, Brian W

Fleckenstein, Monika

Flynn, Jr, Harry W

Fok, Andrew C

Folk, James C
Fortes Filho, Joao B

Foss, Alexander J

Foster, Paul J

Foulds, Wallace S

Fraunfelder, Frederick

Frazer, David G

Friedman, Scott

Fujita, Kyoko

Fulcher, Tim

Gale, Richard

Gallie, Brenda L

Galor, Anat

Gao, Hua

Garg, Prashant

Garg, Sumit

Garg, Sunir

Gariano, Ray

Garudadri, Chandra S

Gaucher, David

Geerling, Gerd

Gemenetzi, Maria

Gentile, Ronald

Georgalas, Ilias

George, Nick

Gerendas, Bianca S

Ghanchi, Faruque D

Ghazi-Nouri, Seyed M

Giansanti, Fabrizio

Giblin, Michael

Gibson, Andrew

Gnanaraj, Lawrence

Goel, Neha

Gogate, Parikshit M

Gogia, Varun

Goldman, Darin

Goldschmidt, Pablo

Goldstein, Michaella

Gole, Glen A

Gomes, André

Gomes, José Álvaro P

Gopinath, Bamini

Gorovoy, Mark

Gottlob, Irene

Goverdhan, Srini

Grigg, John

Grover, Ashok K

Guggenheim, Jeremy

Gunduz, Kaan

Gupta, Aditi

Gupta, Amod

Gupta, Viney

Guthoff, Rudolf

Hall, Anthony

Hall, Edward F

Ham, Don-Il

Hammer, Harold M

Hammond, Chris

Hara, Tsutomu

Harper, Matthew

Harrad, Richard A

Hawkins, Philip

Hayes, Sally

Haylor, Val
Hayreh, Sohan S

Heegaard, Steffen

Heimann, Heinrich

Hemady, Ramzi

Henderson, Bonnie A

Herndon, Leon

Heuer, Dale

Hida, Richard Y

Hirst, Lawrence W

Hjortdal, Jesper O

Holan, Vladimir

Hollo, Gabor

Hood, Donald C

Huang, Shizhou

Humphry, Roger C

Huna-Baron, Ruth

Hunter, David G

Husain, Rahat

Hwang, Young Hoon

Iida, Tomohiro

Innes, William A

Inoue, Makoto

Irion, Luciane

Ishibashi, Tatsuro

Iverson, Shawn M

Jägle, Herbert

Jackson, Timothy L

Jacob, Soosan

James, Nigel J

James, Teifi

Jeng, Bennie $\mathrm{H}$

Jiang, Libin

Jirawuthiworavong, Guy V

Johar, Kaid S

Johnson, Chris A

Johnson, Lenworth

Johnston, Robert L

Jonas, Jost B

Jones, Carole A

Jones, Nicholas P

Jones-Jordan, Lisa

Kalantzis, George K

Kamei, Motohiro

Kannabiran, Chitra

Kaori, Sayanagi

Karacorlu, Murat

Karcioglu, Zeynel A

Karesh, James W

Kasahara, Niro

Katz, Bradley

Kawaji, Takahiro

Kazim, Michael

Keane, Pearse

Kedar, Sachin

Keenan, Jeremy D

Kellner, Ulrich

Kelly, Simon P

Kemp, Ewan G

Kenney, M Cristina

Kenyon, Kenneth

Kersey, James P

Khandhadia, Sam
Khandwala, Mona

Khawaja, Anthony

Kheirkhah, Ahmad

Kiernan, Daniel F

Kim, Chan Yun

King, Anthony J

Kiratli, Hayyam

Kirwan, James

Kishore, Kamal

Knapp, Christopher M

Koh, Adrian $\mathrm{H}$

Kojima, Takashi

Konstantopoulos, Aristides

Koppens, Jo M

Kosmorsky, Greg S

Krishnan, Chandrasekharan

Krohne, Tim U

Kuhn, Ferenc

Kumar, Krishna

Kumar, Mukesh

Kumaramanickavel, Govindasamy

Kupersmith, Mark

Kurian, Mathew

Lagali, Neil

Lai, Chi-Chun

Lai, Timothy Y

Lal Verma, Gopal

Lam, Douglas

Lam, Wai Ching

Landau, Klara

Lane, Carol M

Lauer, Andreas

Lavin, Michael

Lavin, Patrick

Law, Simon K

Lee, Hyunjoo J

Lee, Lawrence

Lee, Vickie

Lemp, Michael A

Leng, Theodore

Lessell, Simmons

Lesser, Robert

Liao, Yaping J

Liarakos, Vasilis S

Liebmann, Jeffrey M

Lim, Lyndell

Lin, Shan

Little, Brian

Liu, CS

Liu, Lance

Liu, Lei

Lloyd, Bender

Lois, Noemi

Luk, Fiona O

Lyons, Christopher J

Mackey, David A

Madge, Simon N

Malhotra, Raman

Mansberger, Steven

Mansour, Ahmad M

Manzouri, Bita

Markham, Richard

Marr, Brian P 
Marsh, Ian B

Mashayekhi, Arman

Mathew, Raeba

Matoba, Alice $Y$

McCannel, Colin A

McCannel, Tara A

McCulley, Timothy J

McKibbin, Martin

McNab, Alan A

McNaught, Andrew I

Melson, Mark R

Menon, Jayshree V

Merayo, Jesús

Meyerle, Catherine B

Michalewska, Zofia

Milenkovic, Vladimir M

Miller, Darlene

Mimura, Tatsuya

Minckler, Don S

Mindel, Joel

Missotten, Tom O

Mohamed, Quresh

Molden, Helen

Mollan, Susan P

Mombaerts, Ilse

Montgomery, Donald M

Moore, Johnny

Morgan, James E

Moshirfar, Majid

Moster, Mark

Mruthyunjaya, Prithvi

Muñoz-Negrete, Francisco J

Mullins, Robert F

Munk, Marion R

Muralidhar, NS

Murdoch, Ian E

Murgatroyd, Helen

Murphy, Paul J

Murray, Desiree

Murray, Philip I

Nagpal, Manish

Nakaya, Naoki

Nanavaty, Mayank A

Nangia, Vinay

Narayanan, Raja

Nehls, Sarah

Nentwich, Martin M

Newsom, Richard S

Ngah, Nor Fariza

Nicolò Massimo

Niederer, Rachael L

Niles, Philip I

Nittala, Muneeswar G

Nolan, Daniel

Nolan, Winnie

Norris, Jonathan $\mathrm{H}$

Nylander, Arthur

O'Connor, Anna

O'Driscoll, Anthony M

Ober, Michael

Oetting, Thomas A

Ogawa, Yoko
Ogura, Yuichiro

Oh, Jaeryung

Ohno-Matsui, Kyoko

Okeefe, Michael

Ong, Chin T

Ophir, Avinoam

Ortega-Larrocea, Gabriela I

Oshima, Yuji

Osman, Amr A

Osoba, Olayinka

Oworu, Gbemi

Packard, Richard

Pagliarini, Sergio

Pal, Dhanajay

Palamar, Melis

Panarelli, Joseph

Pande, Milind

Panjwani, Noorjahan

Papageorgiou, Costas

Pappuru, Rajeev R

Paridaens, Dion

Park, Dong Ho

Pasquale, Louis $\mathrm{R}$

Patel, CK

Patel, Dipika V

Patel, Jignesh I

Pathengay, Avinash

Patton, Niall

Pe'er, Jacob

Pearson, Andrew

Pelletier, Jesse

Phelan, Peter

Phelan, Peter S

Pierscionek, Barbara

Pineles, Stacy

Polkinghorne, Philip J

Potamitis, Theodoros

Prager, Franz

Prasad, Som

Price, Frank

Pulido, Jose S

Querques, Giuseppe

Quigley, Harry A

Raj, Shetal M

Rajak, Saul N

Raman, Rajiv

Ramasubramanian, Aparna

Randleman, J Bradley

Rao, Harsha L

Rapuano, Christopher J

Ratanasukon, Mansing

Read, Scott A

Reck, Anne

Reddy, MA

Reibaldi, Michele

Rekas, Marek

Rennie, Ian

Ressiniotis, Thomas

Ridgway, Elisabeth J

Roberts, Fiona

Rockwood, Edward J

Rosner, Mordechai
Rowe, Fiona J

Ruamviboonsuk, Paisan

Ruiz-Moreno, Jose M

Rundle, Paul A

Russell-Eggitt, Isabelle

Sadrai, Zahra

Saeed, Peerooz

Saha, Konal

Sahin, Afsun

Sahni, Jayashree N

Saito, Wataru

Sakamoto, Taiji

Saleh, George M

Saleh, Maher

Salvi, Sachin M

Sandramouli, Soupramanien

Sandy, Charles

Sarangapani, Susan

Saxena, Sandeep

Sayegh, Rony

Schaumburg, Chris

Schloetzer-Schrehardt, Ursula

Schraermeyer, Ulrich

Scott, Robert

Sehi, Mitra

Seitz, Berthold

Self, Jay E

Sergott, Robert C

Shah, Alpesh R

Shah, Chirag P

Shah, Gauri

Shah, Kalpit P

Shah, Sajani K

Shahid, Humma

Shams, Pari N

Shanmugam, Mahesh

Sharma, Namrata

Shastri, Lajja

Sherwin, Trevor

Sheu, Shwu-Jiuan

Shields, Carol

Shimura, Masahiko

Shortt, Alex J

Shrivastava, Anurag

Shroff, Cyrus M

Shuba, Lesya

Sibony, Patrick

Silvestri, Giuliana

Singh, Arun D

Sloper, John

Smith, George

Smith, Richard

Smith, Scott D

Sobaci, Gungor

Soong, HK

Sorensen, Torben L

Sosne, Gabriel

Spalton, David

Sparrow, John M

Spencer, Fiona A

Stables, Graeme

Stafanous, Sabah

Stalmans, Ingeborg

Stappler, Theodor
Steel, David H

Stewart, Jay M

Stone, Donald U

Stryjewski, Tomasz P

Subak-Sharpe, Ian

Sugar, Alan

Sugar, Joel

Sui, Ruifang

Sullivan, Paul M

Sutphin, John

Swarbrick, Helen

Symeonidis, Chrysanthos

Symes, Richard J

Tambe, Katya A

Tamhankar, Madhura A

Tan, Nikolle W

Tanna, Angelo P

Tao, Yong

Taylor, Robert $\mathrm{H}$

Taylor, Simon

Tekeli, Oya

Teoh, Stephen C

Terai, Naim

Thaller, VT

Thaung, Caroline

Tiffin, Peter

Tint, Naing L

Tong, Louis

Trivedi, Rupal H

Tsaloumas, Marie D

Tsirbas, Angelo

Tu, Elmer Y

Tuft, Stephen J

Tun, Tin A

Tunc, Murat

Ueno, Shinji

Ufret Vincenty, Rafael

Ung, Tsiang

Van den Berg, Tom

Van Stavern, Gregory P

Vandewalle, Evelien

Vaphiades, Michael

Vasavada, Vaishali

Vasavada, Viraj A

Vavvas, Demetrios G

Vernon, Stephen A

Vivian, Anthony J

Vize, Colin

Vohra, Shashi B

Votruba, Marcela

Walker, Sinead

Wallace, Graham

Waterman, Heather

Watts, Patrick

Wavikar, Chandrashekhar

Webster, Andrew R

White, Andrew

Whiteside de Vos, Julia

Whittaker, Karl W

Wiggs, Janey

Williams, Keryn A 
Williams, Michael A Williamson, Tom $\mathrm{H}$

Winder, Stephen

Witkin, Andre J

Wolf, Armin

Wolffsohn, James S

Wollstein, Gadi

Woo, Se Joon
Woodruff, Geoffrey

Wright, Mark

Xing, Dongmei

Yam, Gary H

Yamashiro, Kenji

Yang, Chung-May

Yang, Yit C
Yao, Ke

Yeh, Po-Ting

Yeung, Chi-Kong

Yilmaz, Gursel

Yoon, Kyung-Chul

Yoshida, Shigeo

Younan, Christine

Young, Alvin L
Zambarakji, Hadi

Zamir, Ehud

Zeitz, Oliver

Zhang, Xiaojun

Zhao, Chunming

Zihivov, Andy 